\title{
Value of somatosensory and motor evoked potentials in predicting arm recovery after a stroke
}

\author{
Hilde Feys, Johan Van Hees, Frans Bruyninckx, Rudy Mercelis, Willy De Weerdt
}

Department of Rehabilitation Sciences, Faculty of Physical Education and Physiotherapy, University of Leuven, Belgium

H Feys

W De Weerdt

Department of Neurology, Faculty of Medicine, University of Leuven, Belgium J Van Hees

Department of Physical Medicine and Rehabilitation, University Hospital, Leuven, Belgium

F Bruyninckx

Department of

Neurology, University Hospital, Antwerpen, Belgium

$\mathrm{R}$ Mercelis

Correspondence to: Dr Hilde Feys, Faculty of Physical Education and Physiotherapy, Departmen of Rehabilitation Sciences, Tervuursevest 101, B-3001 Heverlee, Leuven, Belgium email Hilde.Feys@ flok.kuleuven.ac.be

Received 30 June 1999 and in revised form 12 October 1999

Accepted 29 October 1999

\begin{abstract}
Objectives-Prediction of motor recovery in the arm in patients with stroke is generally based on clinical examination. However, neurophysiological measures may also have a predictive value. The aims of this study were to assess the role of somatosensory (SSEPs) and motor (MEPs) evoked potentials in the prediction of arm motor recovery and to determine whether these measures added further predictive information to that gained from clinical examination.
\end{abstract}

Methods-Sixty four patients who had had a stroke and presented with obvious motor deficit of the arm were examined in terms of three clinical variables (motor performance, muscle tone, and overall disability) and for SSEPs and MEPs. Clinical and neurophysiological examinations were done at entry to the study ( 2 to 5 weeks poststroke), and at about 2 months after stroke. Further clinical follow up was conducted at 6 and 12 months after stroke. Results-Neurophysiological measures made in the acute phase were of little use alone in predicting motor recovery of the arm at 2, 6, and 12 months after stroke. At 2 months, the absence of SSEPs and MEPs indicated a very poor outcome. Conversely, if the responses were preserved, a great variation in motor outcome was found. Multiple regression analysis showed that the addition of SSEPs and MEPs to the clinical examination increased the possibility of predicting arm recovery in the long term. In the acute phase, the combination of the motor score and SSEPs were best able to predict outcome. The long term outcome based on variables taken at 2 months, was best predicted through incorporating the three clinical measures and MEPs.

Conclusions-Neurophysiological measures alone are of limited value in predicting long term outcome. However, predictive accuracy is substantially improved through the combined use of both of these measures and clinical variables. (F Neurol Neurosurg Psychiatry 2000;68:323-331)

Keywords: stroke; prognosis; rehabilitation; evoked potentials

Loss of arm function is a common and devastating outcome of stroke. About three quarters of the patients initially show a motor deficit in the upper limb and recovery is generally poor, ${ }^{1-4}$ although a wide variation in outcome has been reported. ${ }^{5}$ An early indication of the level of recovery a patient might achieve would assist in planning appropriate medical and rehabilitation intervention. In the past, prediction of motor recovery of the arm has been based primarily on clinical examination. The degree of initial motor deficit was found to be the most important determinant of motor $^{67}$ and functional ${ }^{1347}$ recovery. There may be other tests which can more accurately predict outcome. The measurement of somatosensory and motor evoked potentials, for example, is an objective and direct method of assessing the integrity of sensory and motor pathways of the CNS. It is possible the evoked potentials may have a predictive value.

Several studies have examined the value of the somatosensory evoked potentials (SSEPs) in the prediction of overall functional recovery ${ }^{8-16}$ or upper limb function. ${ }^{14}{ }^{17-19}$ Most of these indicate that SSEPs correlate well with the subsequent level of disability but two recent publications reported limited prognostic value of these measures. ${ }^{15}{ }^{16}$ The recent development of a non-invasive method of transcranial magnetic stimulation ${ }^{20}$ allows evaluation of the central motor pathways. Since 1989, studies have been conducted to assess the value of motor evoked potentials (MEPs) in patients with stroke, mainly in the prediction of overall functional outcome $\mathrm{e}^{1013151621-27}$ but also of motor $^{1921222528}$ or functional ${ }^{25} 29$ recovery of the upper limb. There is no consensus on the results. Many studies reported MEPs to be prognostic indicators. Timmerhuis et $a l^{16}$ reported that only the evoked potentials measured in the acute stage had predictive value. On the other hand, Catano et $a l^{23}$ concluded that early determination was inconclusive but that evoked potentials taken at 1 month after stroke correlated significantly with outcome. Other authors ${ }^{2126}{ }^{29}$ concluded that motor evoked potentials have no value in predicting outcome of hemiparesis.

The prognostic value of motor versus somatosensory evoked potentials for overall functional recovery has been compared in a few studies. ${ }^{10131516}$ Three studies ${ }^{101516}$ found MEPs to be more sensitive in the prediction of outcome. In the experiment of $\mathrm{Chu}$ and $\mathrm{Wu},{ }^{13}$ motor and somatosensory evoked potentials had different predictive values. The presence of MEPs predicted good functional recovery whereas the absence of SSEPs indicated poor outcome.

Most investigations related single neurophysiological measures to outcome. A few studies assessed the value of neurophysiological measures after factoring in clinical variables 
in a multivariate approach. ${ }^{9}{ }^{16}{ }^{24}$ Results show that an early clinical measurement of the motor deficit was superior to neurophysiological measures. However, adding somatosensory ${ }^{16}$ or motor ${ }^{16}{ }^{24}$ evoked potentials to clinical information improved the precision of prediction.

Several studies on the predictive value of neurophysiological measures have been published; however, as noted above, results were not always convergent. Discrepancies could be due to the inclusion of different patient groups, the variation in outcome measures used, and the divergent methods of quantifying neurophysiological data. In addition, outcome was assessed at various intervals after stroke and the timing of the initial assessment often differed. The sample sizes were small. Most of the studies had 30 or less subjects.

Most of the above studies attempted to predict overall functional recovery rather than specifically recovery of upper limb function. Of those that did focus on the use of SSEPs ${ }^{14}$ 17-19 and MEPs ${ }^{19} 21222528$ in predicting recovery of the upper limb, only three ${ }^{18} 1922$ selected patients on the basis of a motor deficit of the arm. No investigation was found that evaluated the combined predictive value of clinical and neurophysiological measures for upper limb recovery.

It would seem that the role of MEPs and SSEPs in predicting motor recovery of the arm when utilised in conjunction with clinical examination has not been conclusively determined. Therefore the aims of this study were (1) to assess the value of SSEPs and MEPs in the prediction of motor recovery of the upper limb in patients with stroke, and (2) to evaluate the combined predictive value of these measures and a set of clinical variables. The study was confined to patients with a significant motor loss in the arm who were able to participate in a rehabilitation programme. This section of the stroke population is of practical interest for both clinicians and researchers working in the field of rehabilitation.

\section{Methods}

PATIENTS

All patients with stroke who were admitted over a 30 month period to an acute medical ward and referred for rehabilitation, were screened for entry to the study. The diagnosis of stroke was based on clinical history and examination and confirmed by CT. The criteria for inclusion of patients in the trial were: (1) diagnosis of ischaemic brain damage or intracerebral haemorrhage; (2) an obvious motor deficit of the upper limb as evidenced by a score of less than 46 on the Brunnström-Fugl-Meyer ${ }^{30}$ subscale for the upper limb; and (3) sufficient cooperation for clinical and neurophysiological testing. Patients were excluded on the grounds of a subarachnoidal or subdural haemorrhage, a previously completed stroke on the same side, or a prestroke disability affecting arm function. In addition, the use of MEPs precluded patients with implanted metallic devices such as aneurysm clips and cardiac pacemakers, or history of epilepsy. ${ }^{31} \mathrm{~Pa}-$ tients were recruited from several hospitals in the vicinity of Leuven, Belgium (University
Hospitals, Leuven; University Hospital and Rehabilitation Centre Hof ter Schelde, Antwerpen; OLV-hospital, Aalst) all of which implement a similar multidisciplinary approach to rehabilitation.

\section{PROCEDURE}

The subjects were entered into the study between 2 and 5 weeks after onset of the stroke. Baseline clinical and neurophysiological examinations were performed at entry into the study and 6 weeks after the first evaluation (referred to as 2 month measurement). Further clinical follow up was conducted at 6 and 12 months after stroke. Clinical measures included motor performance, muscle tone, and an index of activities of daily living. The tests were performed by physical therapists who were unaware of the results of the neurophysiological examination. Neurophysiological measures consisted of somatosensory and motor evoked potentials and were carried out respectively by the Departments of Neurology and the Departments of Physical Medicine and Rehabilitation. One senior staff neurologist, who was blind to the results of other measurements, interpreted all SSEP recordings. All MEP measurements were interpreted by one senior staff physician who was equally blinded.

The procedures followed were in accordance with the ethical standards of the responsible institutional committee on human experimentation of each hospital. Consent was obtained from all patients participating in the study or from close relatives.

\section{EVALUATION}

\section{Clinical examination}

The section on the upper limb of the Brunnström-Fugl-Meyer test ${ }^{30}$ was used to evaluate motor performance. The test includes items related to movements of the shoulder, elbow, forearm, wrist, and hand, each graded on a two or three point scale. The scores of all subtests can be added up to a total score, varying between 0 and 66. The Ashworth scale ${ }^{32}$ was used to evaluate muscle tone of the internal rotators and adductors of the shoulder, the flexors and extensors of the elbow, the pronators of the forearm, and the flexors of wrist and fingers. A total score of the tone in the seven muscle groups was made (score range: $0-28)$. The Barthel index ${ }^{33}$ was used as a measure of overall disability (score range: 0-100). All clinical measures met the criteria of reliability ${ }^{34-36}$ and validity. ${ }^{3033} 37$

\section{Neurophysiological examination}

The examination of the SSEPs was performed by stimulation of the left and right median nerves at the wrist with at least a four channel system (Nomad Tracor, Mystro, or Nicolet Viking II apparatus). The stimulus rate was 5 $\mathrm{Hz}$ and the stimulus intensity that elicited a visible twitch of the thumb muscles was used. Scalp placement of the electrodes was in accordance with the 10-20 International System. Silver-silver chloride electrodes were placed over Erb's point, the seventh cervical vertebra, and contralateral somatosensory 
areas ( $2 \mathrm{~cm}$ behind $\mathrm{C} 3$ and $\mathrm{C} 4)$. The reference electrode was placed at Fz. At least two trials were performed to ensure the reproducibility of the evoked response components. The evoked potentials were obtained by averaging the recordings after 500 stimuli. Responses were filtered through a bandpass of 5 to $1500 \mathrm{~Hz}$. The absolute latencies for N9 (Erb's point) and N19 (contralateral cortex), the N9-N19 interpeak latency, the side by side differences of the latencies, and the peak to peak amplitude of the N19/P22 complex were determined.

Magnetic motor stimulation was carried out by a Magstim Model 200 or Cadwell MES 10 stimulator with a $9 \mathrm{~cm}$ diameter circular coil. The coil was placed over the vertex, slightly away from the midline to activate the motor cortex and laterally over the $\mathrm{C} 7$ spinal process to activate the ventral roots. Stimuli were given on both sides with increasing intensity until potentials of the largest amplitude and shortest latency were obtained. The stimulus intensity was increased to $100 \%$ before a response was considered absent. During stimulation of the motor cortex, patients were asked to make a slight contraction of the target muscle or to contract the healthy limb in cases of severe paresis. Motor responses evoked by transcranial magnetic stimulation were recorded from the abductor pollicis brevis muscle by surface electrodes using a conventional EMG system. For each patient, peripheral nerve stimulation was also performed to obtain the optimal electrode positioning by checking the amplitude and to exclude carpal tunnel syndrome or peripheral neuropathy by checking the motor nerve conduction velocity. The central motor conduction time, calculated as the latency difference between responses to stimulation of the motor cortex and the ventral roots and the between side difference in the central motor conduction time were determined. The amplitude of the muscle response after cortical stimulation was measured from peak to peak.

All somatosensory and motor responses were classified into three categories on the basis of latencies and amplitude. The first category included normal responses. Waveforms with delayed central conduction times, or a significant interside latency difference, or a left-right difference in amplitude of $50 \%$ or more were designated as category II (referred to as a pathological response). The SSEPs with a deviating configuration were also classified in this group. Subjects in whom no potentials could be evoked were allocated to category III. The normal values, established by Chiappa ${ }^{38}$ and Eisen and Shtybel, ${ }^{39}$ were used as references for SSEPs and MEPs respectively.

DATA ANALYSIS

Descriptive statistics were used to describe the patients' characteristics and to document the results of SSEPs and MEPs.

The prognostic values of SSEPs and MEPs were first examined using univariate statistics. Relations between neurophysiological measures and motor outcome were explored by scatter plots and motor outcome was compared between the different categories of the evoked potentials by means of analysis of variance (ANOVA) and Student's $t$ test.

A multivariate approach was used to assess if the evoked potentials had an additional predictive value over the clinical examination. The selection of the clinical variables was based on previous studies, ${ }^{167}$ in which motor recovery of the arm was predicted from many clinical variables. Five predictor variables were selected: SSEPs, MEPs, motor recovery, overall disability, and muscle tone. Multiple regression analyses were carried out to make the following predictions: (1) level of motor recovery at 2, 6 , and 12 months after stroke from predictor variables measured at baseline, and (2) level of motor recovery at 6 and 12 months from predictor variables evaluated at 2 months after the onset of stroke.

Regression analysis was first performed including only one variable in the model to establish the individual contribution of each predictor variable. To determine which combination of predictor variables led to the best predictive model, variables were omitted from the model step by step, on the basis of significance testing of the regression coefficients and the $\mathrm{R}^{2}$ selection method combined with the Mallows $\mathrm{C}(\mathrm{P})$ statistic. The $\mathrm{R}^{2}$ variable selection method computes the $R^{2}$ of all possible models for all subset sizes. The $\mathrm{C}(\mathrm{P})$ statistic is a measure indicating evidence of bias towards an incompletely specified model (too few variables) or towards an overspecified model (too many variables). ${ }^{40}$ The model with the highest $\mathrm{R}^{2}$ and optimum $\mathrm{C}(\mathrm{P})$ statistic with all the predictor variables significant $(p<0.05)$ was retained for each of the predictions. For all selected models, the assumptions underlying multiple regression were tested by inspecting the distribution of the error term and the data set was examined for possible problems involving outliers, influential points, and multicollinearity. ${ }^{40}$

All statistical procedures were performed with the SAS system.

The present study was part of a larger study investigating the effect of a therapeutic intervention on motor recovery of the upper limb in stroke subjects. ${ }^{41}$ Hence, the factor "group", referring to an experimental and control condition, and the interactions of this factor with other predictor variables were added to the model. Multiple regression analyses showed no interactions between the factor group and other predictive variables and the factor group was not found to be significant. Therefore, the results are based on the total sample.

\section{Results}

PATIENTS

Sixty four patients fulfilled the criteria for inclusion in the study of SSEPs at baseline and 61 at 2 months. Of these, 50 and 43 were able to undergo measurement of MEPs at baseline and 2 months respectively. Three and six patients were lost to follow up at 6 and 12 months respectively because of death, recurrence of stroke, precarious medical condition, or refusal. 
The mean age of patients $(n=64)$ was 63.69 years (SD 11.34) with a range of 38 to 88 . The mean time interval between the occurrence of stroke and the baseline measurement was 22.08 days (SD 6.49; range 13-37). There were 37 men and 27 women. Thirty eight patients had a left hemiparesis, 26 a right hemiparesis. The cause of stroke was ischaemic in 61 patients. Three patients had an intracerebral haemorrhage. The initial motor scores on the Brunnström-Fugl-Meyer test varied between 4 and 41 with a mean of 12.94 (SD 10.16). The mean value on the Barthel index at entry into the study was 45.47 (SD 18.72; range 10-95).

NEUROPHYSIOLOGICAL MEASURES

At baseline $(\mathrm{n}=64)$, SSEPs were normal in 10 $(16 \%)$ patients, pathological in $30(47 \%)$, and absent in $24(37 \%)$. These figures were respec-
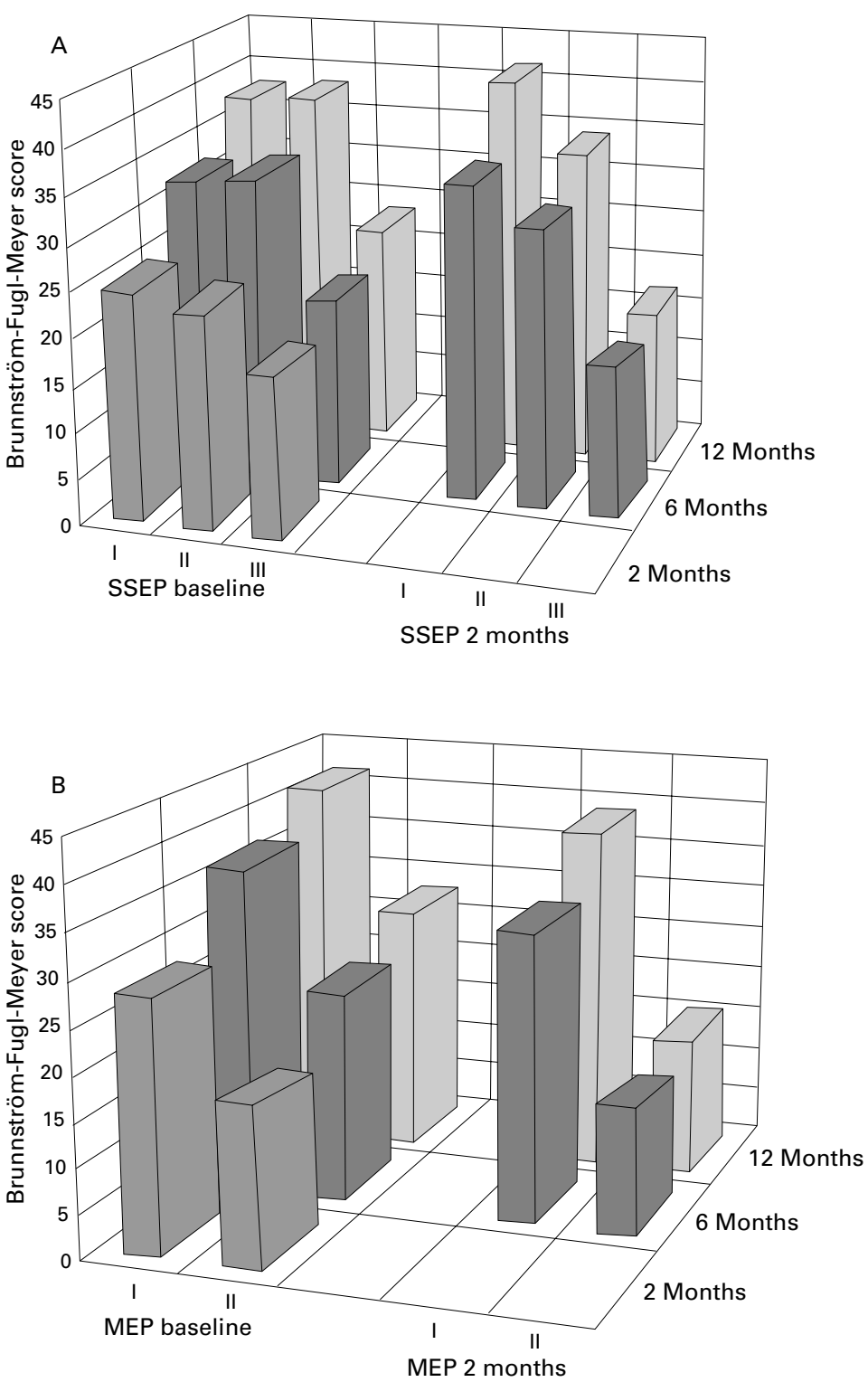

Figure 1 Mean scores on the Brunnström-Fugl-Meyer test at 2, 6, and 12 months after stroke by SSEP $(A)$ and MEP $(B)$ category measured at baseline (left), and mean scores on the Brunnström-Fugl-Meyer test at 6 and 12 months by SSEP $(A)$ and $M E P(B)$ category measured at 2 months after the onset of stroke (right). For SSEP: I=normal response; $I I=$ pathological response; $I I I=$ absent response. For $M E P: I=$ present response; $I I=$ absent response. tively $13(21 \%), 30(49 \%)$, and $18(30 \%)$ at the 2 month assessment $(n=61)$. Transcranial magnetic stimulation performed at baseline $(\mathrm{n}=50)$ evoked a normal response in only one patient. Twelve (24\%) patients showed a pathological response and no response was obtained in $37(74 \%)$. At the second evaluation $(n=43)$, responses were normal in two $(4.5 \%)$ patients, pathological in $23(53.5 \%)$, and absent in $18(42 \%)$.

At baseline, 50 patients were assessed for both SSEPs and MEPs. Of the 29 showing a SSEP response, 10 had absent MEP responses. Three out of 18 patients with absent SSEPs had a preserved MEP response. On the second test, the two neurophysiological measurements were performed on 42 patients. Of the 26 patients with SSEPs present, eight had no MEP response. On the other hand, six of the 16 patients without a SSEP response showed a MEP response.

PREDICTION OF MOTOR RECOVERY

Somatosensory and motor evoked potentials

Figure 1 A depicts the mean scores on the Brunnström-Fugl-Meyer test at the different time intervals for each category of the SSEPs measured at baseline and at 2 months. An ANOVA showed no significant differences between the SSEP categories with regard to the mean 2 month Brunnström-Fugl-Meyer scores $(F=1.23, \mathrm{p}=0.30)$. At 6 and 12 months, there were significant differences between the Brunnström-Fugl-Meyer scores of the SSEP categories, whether the scores were categorised according to baseline SSEP measures (6 months: $F=4.39, \mathrm{p}=0.02 ; 12$ months: $F=3.97$, $\mathrm{p}=0.02)$ or 2 month SSEP measures (6 months: $F=7.30, \mathrm{p}=0.002 ; 12$ months: $F=7.62$, $\mathrm{p}=0.001)$. In baseline SSEP categories, differences between the Brunnström-Fugl-Meyer scores were only significant between category II and III. If the 2 month SSEP categories were utilised, mean scores were significantly higher in categories I and II versus category III.

One patient at baseline and two patients at 2 months showed a normal response to transcranial magnetic stimulation. The patients' responses were therefore classified into two categories, with category I corresponding to a response being present and category II to an absent response. Figure $1 \mathrm{~B}$ depicts the mean values of Brunnström-Fugl-Meyer scores at different time intervals for the two categories of MEPs, measured at baseline and at 2 months. Student's $t$ tests showed significant differences between the Brunnström-Fugl-Meyer scores of both categories of MEPs measured at baseline, at 2 months $(t=2.21, \mathrm{p}=0.03), 6$ months $(t=2.51, \mathrm{p}=0.02)$, and 12 months $(t=2.17$, $\mathrm{p}=0.04)$. Differences were even more pronounced when 2 month MEP categorisation was used ( 6 months: $t=5.41, \mathrm{p}=0.0001 ; 12$ months: $t=5.12, \mathrm{p}=0.0001)$.

Although there were obvious differences between the mean scores on the BrunnströmFugl-Meyer test, the SDs were large. Variation coefficients, calculated as the SD divided by the mean and multiplied by 100 , varied between $40 \%$ and $81 \%$. Therefore, a more 

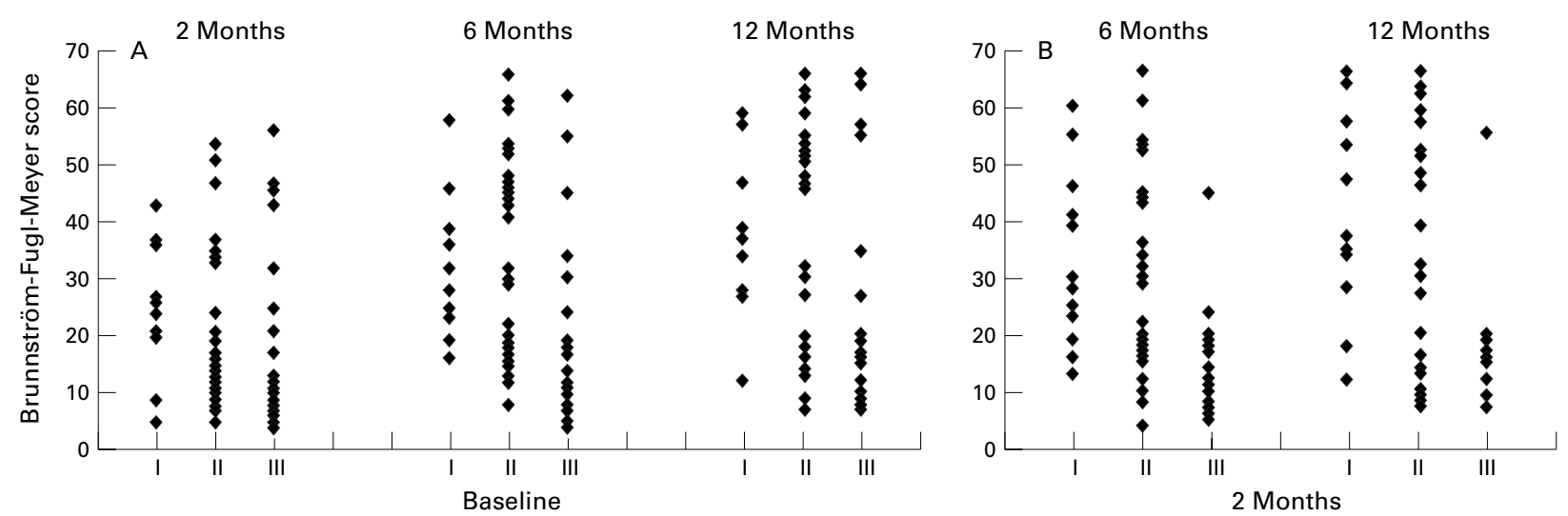

Figure 2 Scatter plot of Brunnström-Fugl-Meyer scores at 2, 6, and 12 months after stroke by SSEP category measured at baseline (A), and Brunnström-Fugl-Meyer scores at 6 and 12 months after stroke by SSEP category measured 2 months after the onset of stroke (B). I=normal response; $I I=$ pathological response; $I I I=$ absent response.
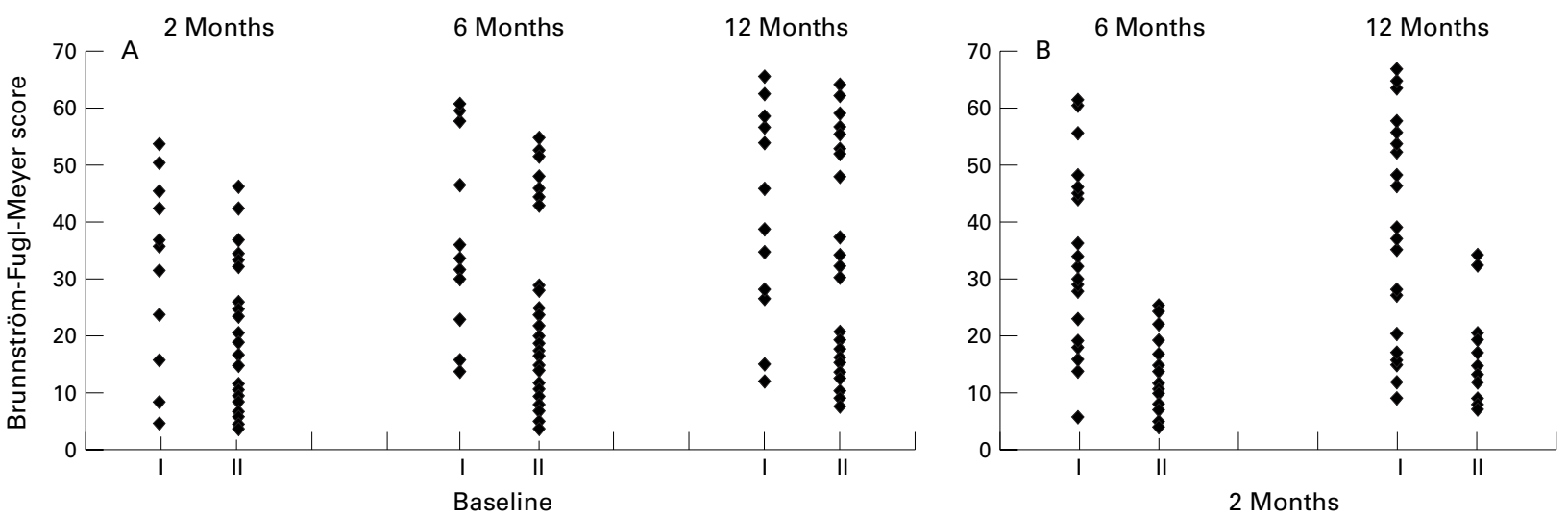

Figure 3 Scatter plot of Brunnström-Fugl-Meyer scores at 2, 6, and 12 months after stroke by MEP category measured at baseline (A), and Brunnström-Fugl-Meyer scores at 6 and 12 months after stroke by MEP category measured 2 months after the onset of stroke (B). I=present response; $I I=$ absent response.

detailed analysis of the results was performed. The individual Brunnström-Fugl-Meyer motor scores at 2, 6, and 12 months after stroke were plotted for the several categories of SSEPs (fig 2) and MEPs (fig 3). If the baseline SSEPs were used as the basis for categorisation, motor scores at 2, 6, and 12 months after stroke were very variable, whether patients had a category I, II, or III response. For measurement of SSEPs at 2 months, a variable outcome was found for patients belonging to category I or II.

Table $1 R^{2}$ values obtained by regression analysis for the prediction of motor recovery of the arm at 2, 6 , and 12 months post-stroke from five single predictor variables measured at baseline, and for the prediction of motor recovery of the arm at 6 and 12 months post-stroke from five single predictor variables measured at 2 months after the onset of stroke

\begin{tabular}{llll}
\hline & Predictors & At baseline & At 2 months \\
\hline Outcome at 2 months & Motor performance & $\mathrm{n}=50$ & $70.25^{\star \star \star}$ \\
& Overall disability & $17.81^{\star \star}$ & \\
& Muscle tone & 2.11 & \\
& SSEP & 7.22 & \\
& MEP & $9.27^{\star}$ & \\
Outcome at 6 months & & $\mathrm{n}=48$ & $\mathrm{n}=40$ \\
& Motor performance & $52.09^{\star \star \star}$ & $74.81^{\star \star \star}$ \\
& Overall disability & $22.79^{\star \star \star}$ & $36.72^{\star \star \star}$ \\
& Muscle tone & 0.80 & $10.19^{\star}$ \\
& SSEP & $20.24^{\star \star \star}$ & $24.29^{\star \star \star}$ \\
Outcome at 12 months & MEP & $12.07^{\star}$ & $35.83^{\star \star \star}$ \\
& Motor performance & $49.41^{\star \star \star}$ & $\mathrm{n}=37$ \\
& Overall disability & $22.24^{\star \star \star}$ & $38.30^{\star \star \star}$ \\
& Muscle tone & 1.29 & $35.92^{\star \star \star}$ \\
& SSEP & $21.86^{\star \star \star}$ & $14.08^{\star}$ \\
& MEP & $9.88^{\star}$ & $37.76^{\star \star}$ \\
& & & \\
\hline
\end{tabular}

${ }^{\star} \mathrm{p}<0.05 ;{ }^{\star \star} \mathrm{p}<0.01 ;{ }^{\star \star \star} \mathrm{p}<0.001$.
However, for all but one patient in category III, motor scores were lower than 24 at 6 months and lower than 20 at 12 months after stroke. Similar outcomes were found for the motor evoked potentials.

\section{Somatosensory and motor evoked potentials in relation to clinical variables}

For the purposes of regression analysis, SSEP and MEP scores were (based on previous results) dichotomised in a non-responsive (score 0) versus a responsive (score 1) group. The responsive group included patients with normal and pathological responses. These variables were combined with the three selected clinical variables. The results are based on fewer patients as regression analysis requires a score for all predictor variables. The number of patients used for each analysis is indicated in the tables.

Table 1 shows the $\mathrm{R}^{2}$ values obtained by regression analysis in predicting motor recovery at the different time intervals from each of the five predictor variables separately. The $\mathrm{R}^{2}$ values represent the percentage of variance in the outcome variable accounted for by each predictor variable. The highest amount of variance was explained by the motor score at an earlier stage; the $\mathrm{R}^{2}$ values varied between $49 \%$ and $75 \%$. The second most important individual predictor of motor outcome was the Barthel index. The maximum explained vari- 
ance was $37 \%$. The $\mathrm{R}^{2}$ values for muscle tone were not significant when measured at baseline but were significant, although low, at 2 months. The $\mathrm{R}^{2}$ values of SSEPs and MEPs measured at baseline were lower than $10 \%$ for predicting outcome at 2 months. At baseline, the value of SSEPs in predicting the level of motor recovery at 6 and 12 months was better than the MEPs. The explained variance from SSEPs was slightly more than $20 \%$. When measured at 2 months, MEPs explained respectively $36 \%$ and $37 \%$ of the variance of motor outcome at 6 and 12 months. The explained variance by SSEPs was 12 to $13 \%$ lower.

Table 2 shows the results of the multiple regression analyses used to predict motor recovery at different time intervals using the three clinical and two neurophysiological measures taken at baseline and at 2 months. For each prediction, the best fitting model is presented, including the $\mathrm{R}^{2}$, the significant predictor variables with corresponding regression coefficients, hypothesis testing, and added $\mathrm{R}^{2}$ or uniqueness index. The uniqueness index is the percentage of variance in the criterion variable accounted for by that predictor, beyond the variance accounted for by other predictor variables. It is an indicator of the relative importance of selected variables to the prediction model.

The most parsimonious model in predicting motor recovery at 2 months from measurements taken at baseline included two clinical variables: motor performance and muscle tone. Combined they explained $74 \%$ of the variance of the outcome measure. The motor score accounted for the largest proportion of variance. Motor performance and SSEPs, measured at baseline, accounted for $60 \%$ and $58 \%$ of the explained variance at 6 and 12 months. Again, the motor score contributed most to the prediction equation, but a substantial amount of variance $(8 \%)$ was also explained by the SSEPs. The MEPs were not retained as a significant variable. Prediction of the level of motor recovery at 6 and 12 months based on measurements sampled at 2 months led to 2 analogue models. Respectively $85 \%$ and $81 \%$ of the variability in outcome was accounted for by using a combination of motor performance, MEPs, overall disability, and muscle tone. Once more, the motor score showed a conspicuous unique contribution. The MEP measurement was the second most important predictor and added $5 \%$ to $6 \%$ to the explained variance.

Finally, patients were classified into four groups based on the motor score and SSEPs at baseline and motor score and MEPs at 2 months (fig $4 \mathrm{~A}$ and $\mathrm{B}$ ). The first category included patients with a moderate motor deficit (Brunnström-Fugl-Meyer: 14 to 46) and a present neurophysiological response. Subjects with a moderate motor deficit and absent response were assigned to category II. The next two categories included the patients with a severe motor deficit. In categories III and IV, the neurophysiological response was respectively present and absent. Figure $4 \mathrm{~A}$ shows the Brunnström-Fugl-Meyer scores at 6 and 12 months for each of the four categories based on the motor score and SSEPs, measured at baseline. Patients with an initial moderate motor deficit performed well at 6 and 12 months, whether the SSEPs were present or not. Motor outcome was very inconsistent in patients with an initial severe motor deficit and preserved SSEPs. However, in all but one of the patients with a severe motor deficit combined with absent SSEPs, recovery was poor. Fig $4 \mathrm{~B}$ shows a scatter plot of the classification of motor performance in conjunction with MEPs measured at 2 months after stroke. Patients with a moderate motor deficit and present MEPs, showed a more favourable outcome but there were some exceptions. The three patients with an absent MEP did not recover well. Motor scores were poor in all patients showing

Table 2 Best models obtained by multiple regression analyses for the prediction of motor recovery of the arm at 2, 6 , and 12 months post-stroke from five predictor variables measured at baseline, and for the prediction of motor recovery of the arm at 6 and 12 months post-stroke from five variables measured at 2 months after the onset of stroke

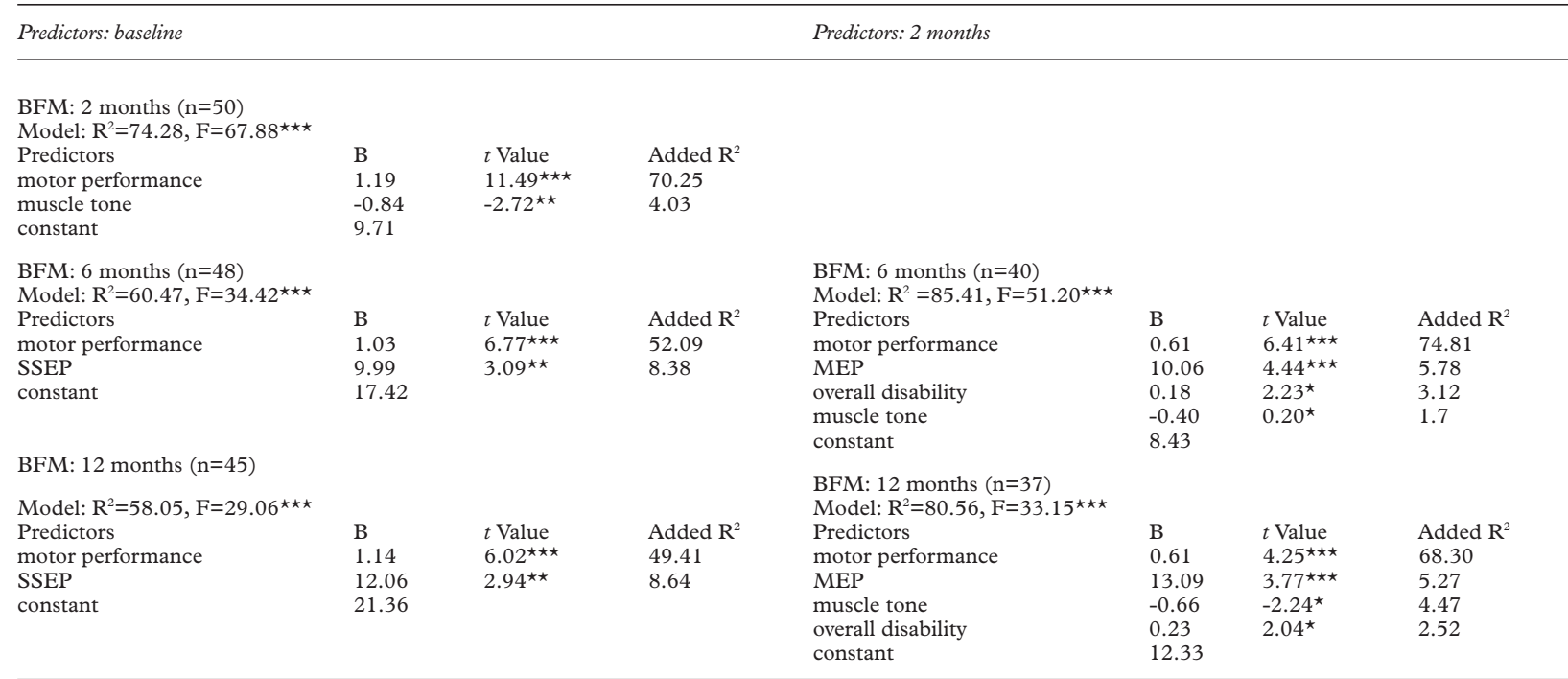



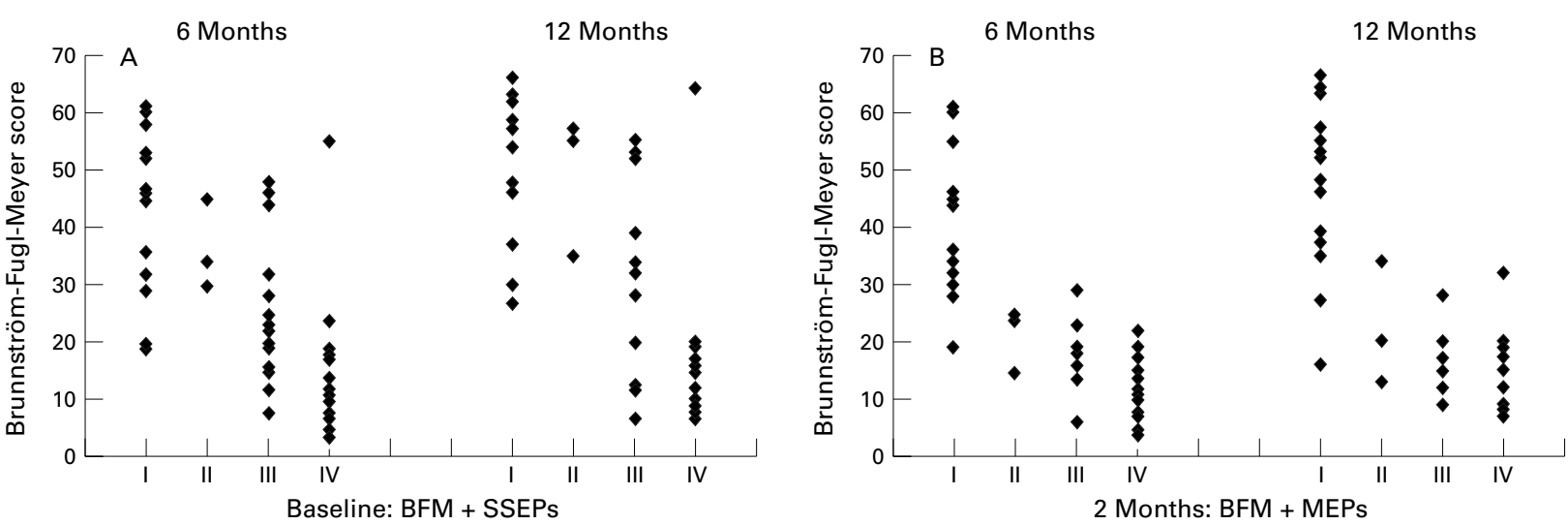

Figure 4 Scatter plot of Brunnström-Fugl-Meyer scores at 6 and 12 months after stroke from Brunnström-Fugl-Meyer scores and SSEPs measured at baseline (A) and from Brunnström-Fugl-Meyer scores and MEPs measured 2 months after stroke (B). I=moderate deficit and present SSEP or MEP; $I I=$ moderate deficit and absent SSEP or MEP;III=severe deficit and present SSEP or MEP;IV=severe deficit and absent SSEP or MEP.

a severe motor deficit at 2 months, regardless of whether MEPs were preserved or not.

\section{Discussion}

Somatosensory and motor evoked potentials assess anatomically distinct pathways. Consequently they may be affected independently after stroke, as evidenced by the present study. In the acute phase, only one out of 50 patients showed a normal response to transcranial magnetic stimulation and responses were absent in most patients (74\%). After 2 months, only two patients had normal MEP responses. This could be attributed to the fact that a criterion for admission in the study was the diagnosis of stroke with a motor deficit in the arm. In general, responses were better preserved for the SSEPs but no difference in motor outcome could be found between patients with normal or pathological SSEPs. Based on these results, the predictive values of both SSEPs and MEPs were further analysed using a classification of absent versus present responses.

The mean level of motor recovery at 6 and 12 months after stroke was significantly different between patients with absent and present responses for SSEPs and MEPs, whether they were measured at baseline or at 2 months. This might suggest some association between neurophysiological measures and motor outcome. However, inspection of individual data shows that in the acute phase, neurophysiological measures alone were of little help in predicting motor recovery up to 12 months after stroke. Variable motor outcome was also found when 2 month responses were utilised. However, the absence of SSEPs or MEPs did indicate poor recovery. This finding was similar to some earlier studies, ${ }^{13}{ }^{17}$ but disagreed with others, ${ }^{911} 13$ which reported that the preservation of evoked potentials was a strong indicator of good recovery whereas absent responses led to a variable outcome. The amount of variance of the outcome explained by the neurophysiological measures, although clearly higher if examined at 2 months, indicated the limited predictive value of these measures alone. Also Arac et $a l^{11}$ and Zgur $e t a l^{29}$ reported no predictive value of MEPs measured in the acute phase. However, these results contradict most previ- ous research for several possible reasons. Many studies limited analysis to comparison of mean scores or correlation coefficients and although significant differences or small associations may have been reported, these do not imply accurate prediction. In addition, the patient populations and outcome measures (usually overall functional recovery) of these studies may not be equated with the present study.

In multivariate analyses, the motor score was consistently retained as the main predictive factor and accounted for most of the variance in all selected models. This was in accordance with previous studies. ${ }^{1367}$ For the prediction from baseline to 2 months, neither of the two neurophysiological measures were retained in the model. In all other prediction models the MEPs or SSEPs accounted for a greater amount of variance than the two clinical predictor variables, overall disability and muscle tone. In the acute phase, the combination of motor performance and SSEPs best predicted upper limb outcome at 6 and 12 months after stroke. The MEPs did not add significant information. Possibly this is a reflection of the closer association between motor function and MEPs compared with motor function and SSEPs. Point-biserial coefficients equalled 0.55 and 0.23 respectively (coefficients were not mentioned in the results section). Consequently, the MEPs may not have added a further significant unique contribution. The model which best predicted 6 and 12 month outcome based on variables measured at 2 months, included the three clinical variables and MEPs.

A scoring system combining the motor deficit and the presence or absence of SSEPs or MEPs (fig 4) illustrates the prognostic information if neurophysiological measures are added to clinical measures in the model. In the acute stage, an initial moderate motor score predicted a favourable long term outcome. A low initial motor score led to a variable outcome. However, if a low initial score was combined with absent SSEPs, this indicated poor outcome. At 2 months, a low motor score alone was an accurate predictor in those patients with a severe motor deficit but less accurate for patients with a moderate deficit. 
For the patients, with a moderate deficit, absent MEPs indicated an unfavourable outcome as well. In summary, absent SSEPs and MEPs both gave additional information to the clinical examination.

The SSEPs were retained as a significant predictor for long term outcome and improved the predictive accuracy with about $8 \%$ when measured at baseline. In other studies, ${ }^{17}$ clinical measures of sensory loss were also retained as significant predictors. These findings stress the prognostic value of sensory impairment in outcome, especially when measured in the acute phase. The SSEPs can be tested in patients with communication deficits due to aphasia or in patients whose mental state does not permit an accurate clinical examination. If predictive models are to be used in practice, it is important that they can be applied to a maximum number of patients.

To the best of our knowledge, Timmerhuis et $a l^{16}$ were the only authors who evaluated the prognostic value of SSEPs and MEPs in combination with a clinical examination. However, the results of their study are not comparable as the outcome measure and time frame of their study were different. They predicted the Barthel index at 3 months after stroke, from measurements taken within the first week and at 6 weeks after stroke onset.

The best prediction of long term outcome from the acute phase was obtained by a combination of motor performance and the SSEPs. The explained variance at 6 and 12 months was $60 \%$ and $58 \%$ respectively. For those patients with severe motor deficit and preserved SSEPs, the prediction was not accurate. The accuracy of prediction can be substantially improved by making predictions step by step. An initial prediction of 2 month outcome can be made in the acute phase exclusively on the basis of clinical measures. The long term prognosis (12 months) can be made at 2 months based on the three clinical measures and MEPs. Application of this strategy to our results led to an explained variance of between $74 \%$ and $81 \%$. Prediction equations of the best models are summarised in the appendix.

Most of the predictive value of the models seemed to derive from relatively simple clinical measures which can be used during routine clinical evaluation of a patient. From a pragmatic point of view, it could be argued that it may not be worthwhile to extend prognostic evaluations to include more complex laboratory tools such as neurophysiological testing. In the absence of facilities, reliable prognostic information can be obtained from clinical examination, provided that predictions are made step by step in time. However, long term prediction from exclusively clinical examination in the acute phase showed moderate accuracy $( \pm 50 \%)$ and the added prognostic value provided by SSEPs $( \pm 8 \%)$ would clearly be beneficial. The good prognostic value of SSEPs in patients with a very poor motor function in the arm in the acute phase leads to the recommendation that this measure be applied to this subgroup. Analogous but less pronounced examination of MEPs at 2 months may provide additional valuable information in patients with a moderate deficit of the arm.

In this study, it has been shown that a reasonably accurate prediction of the level of motor recovery of the upper limb in patients with stroke can be made from measurements sampled in the acute phase. This prediction can further be improved after a short period of rehabilitation. Two months after stroke seems the appropriate time to evaluate the results of an intensive period of rehabilitation, especially with regard to the amount of therapeutic resources allocated under strict cost efficiency criteria. In addition, specific patient groups were identified for whom a very precise prognosis could be made. This information is valuable in rehabilitation planning. It helps therapists to set realistic goals for the treatment of the upper limb in each patient individually and to determine how much rehabilitation effort should be spent on the upper limb in the group of patients who show an extremely poor prognosis. The results of this study can also be translated into practical prognostication in experimental studies. Homogeneous prognostic groups can be identified in controlled trials and thus fewer patients will be needed to achieve the necessary power. This should make it possible to identify the most adequate treatment modalities for subgroups of patients. Researchers may be encouraged to critically appraise existing or emerging therapeutic interventions directed at recovery of the affected arm in patients with stroke.

In this study, motor rather than functional recovery was chosen as an outcome measure. Motor recovery is a more direct measure of the severity of brain damage. The motor scale used is sensitive to change and does not suffer from a bottom effect. Functional recovery will undoubtedly be more meaningful to patients and other professionals in stroke care. However, De Weerdt and Harrison ${ }^{37}$ indicated a very high relation $\left(r_{\mathrm{s}}>0.90\right)$ between motor and functional recovery of the upper limb after stroke. In addition, prediction of motor recovery of the affected arm in patients with stroke has proved to be more accurate in comparison with functional recovery. ${ }^{7}$

It can be concluded that in the acute phase, neurophysiological measures alone are not helpful in predicting motor recovery of the upper limb in patients with stroke and their value is limited even at 2 months. However, clear benefit is obtained when neurophysiological measures are used in combination with clinical examination. To further generalise predictive measures, it is suggested that similar studies be conducted across centres with different approaches towards rehabilitation.

We express our gratitude to the heads of departments and all members of staff of the participating centres for their collaboration. We acknowledge Professors W Pelemans, R Lysens, $\mathrm{H}$ Carton, R Verhaeghe, and M Driessens, and Drs P Cras, J Broeckx, and $G$ Vermeersch. We are also grateful to the physical therapists, G Nuyens, S Blankart, B Sallin, and A Hare physical therapists, G Nuyens, S Blankart, B Sallin, and A Hartmeier for carrying out the blind clinical assessments. Special thanks are offered to Professors M Vuylsteke and E Lesaffre, and Dr D Belmans for their advice on statistical analyses and to J Jelsma, for carefully reviewing the manuscript. This work was supported by a grant from the Nationale Vereniging tot Steun aan Gehandicapte Personen (NVSG). 
Appendix: regression equations for predicting motor recovery of the arm at 2,6 , and 12 months after stroke from two measurement
Baseline to 2 months:

Baseline to 2 months:
motor recovery $=9.71+1.19^{\star}$ (motor performance $)-0.84^{\star}$ motor recovery
(muscle tone)

Baseline to 6 months:

motor recovery $=17.42+1.03^{\star}($ motor performance $)+9.99^{\star}$ (SSEPs)

Baseline to 12 months:

motor recovery $=21.36+1.14^{\star}($ motor performance $)+12.06^{\star}$ (SSEPs)

2 months to 6 months:

motor recovery $=8.43+0.61^{\star}$ (motor performance $)+10.06^{\star}$ (MEPs) $-0.40^{\star}$ (muscle tone) $+0.18^{\star}$ (overall disability)

2 months to 12 months:

motor recovery $=12.33+0.61^{\star}($ motor performance $)+13.09^{\star}$ (MEPs) $-0.66^{\star}$ (muscle tone) $+0.23^{\star}$ (overall disability)

Motor recovery=the predicted score on the BrunnnströmFugl-Meyer test, arm section; the score can vary between 0 Fugl-M

Motor performance=the actual score on the BrunnnströmMotor performance $=$ the actual score on the Brunnnström-
Fugl-Meyer test, arm section; the score can vary between 0

and 66

Muscle tone=the sum score of seven critical muscles, assessed with the Ashworth scale; the score can vary between 0 and 28 . Overall disability=the score on the Barthel Index; the score can vary between 0 and 100 .

SSEPs - two scores: $0=$ absent response; $1=$ present response.

MEPs - two scores: $0=$ absent response, $2=$ present response

1 Wade DT, Langton Hewer R, Wood VA, et al. The hemiplegic arm after stroke: measurement and recovery. 7 Neuro Neurosurg Psychiatry 1983;46:521-24.

2 Parker VM, Wade DT, Langton Hewer R. Loss of arm function after stroke: measurement, frequency, and recovery. In Rehabil Med 1986;8:69-73.

3 Olsen TS. Arm and leg paresis as outcome predictors in stroke rehabilitation. Stroke 1990;21:247-51.

4 Nakayama H, Jörgensen HS, Raaschou HO, et al. Recovery of upper extremity function in stroke patients: the Copenhagen stroke study. Arch Phys Med Rehabil 1994;75:394-8.

5 Heller A, Wade DT, Wood VA, et al. Arm function after stroke: measurement and recovery over the first 3 months. stroke: measurement and recovery over the first 3

6 Gowland C. Predicting sensorimotor recovery following stroke rehabilitation. Physiotherapy Canada 1984;36:31320.

7 De Weerdt W, Lincoln NB, Harrison MA. Prediction of arm and hand function recovery in stroke patients. Int $\mathcal{F}$ Rehabi Res 1987;10:110-2.

8 Zeman BD, Yiannikas C. Functional prognosis in stroke: use of somatosensory evoked potentials. F Neurol Neurosurg Psychiatry 1989;52:242-7.

9 Chester CS, McLaren CE. Somatosensory evoked response and recovery from stroke. Arch Phys Med Rehabil 1989;70 $520-5$.

10 Macdonell RAL, Donnan GA, Bladin PF. A comparison of somatosensory evoked and motor evoked potentials in stroke. Ann Neurol 1989;25:68-73.

11 Gott PS, Karnaze DS, Fisher M. Assessment of median nerve somatosensory evoked potentials in cerebral nerve somatosensory evoked pote

12 Kovala T. Prognostic significance of somatosensory potentials evoked by stimulation of the median and posterior
tibial nerves: a prospective 1 year follow up study in patients with supratentorial cerebral infarction. Eur Neurol 1991;31:141-8

$13 \mathrm{Chu} \mathrm{N}$, Wu T. Motor response patterns and prognostic value of transcranial magnetic stimulation in stroke patients. In: Lissens MA, ed. Clinical applications of magnetic transcranial stimulation. Leuven: Peeters Press, 1992:12745.

14 Keren O, Ring H, Solzi P, et al. Upper limb somatosensory evoked potentials as a predictor of rehabilitation progress in dominant hemisphere stroke patients. Stroke 1993;24: 1789-93.

15 Péréon Y, Aubertin P, Guihéneuc P. Prognostic significance of electrophysiological investigations in stroke patients: somatosensory and motor evoked potentials and sympathetic skin response. Neurophysiol Clin 1995;25:146-57.

16 Timmerhuis TPJ, Hageman G, Oosterloo SJ, et al. The prognostic value of cortical magnetic stimulation in acute
middle cerebral artery infarction compared to other parameters. Clin Neurol Neurosurg 1996;98:231-6.
17 La Joie WJ, Reddy NM, Melvin JL. Somatosensory evoked potentials: their predictive value in right hemiplegia. Arch Phys Med Rehabil 1982;63:223-6.

18 Hendricks HT, Pasman JW, Mulder T, et al. Value of somatosensory evoked potentials for the prediction of motor recovery of the upper extremity after cerebral infarction. Fournal of Rehabilitation Sciences 1994;7:3-8.

19 Hendricks HT, Hageman G, Van Limbeek J. Prediction of recovery of upper extremity paralysis after stroke by measuring evoked potentials. Scand F Rehabil Med 1997:29:1559 .

20 Barker AT, Freeston IL, Jalinous R, et al. Magnetic stimulation of the human brain and peripheral nervous system: an introduction and the results of an initial clinical evaluation. Neurosurgery 1987;20:100-9.

21 Arac N, Sagduyu A, Binai S, et al. Prognostic value of transcranial magnetic stimulation in acute stroke. Stroke 1994; 25:2183-6.

22 Rapisarda G, Bastings E, Maertens de Noordhout A, et al. Can motor recovery in stroke patients be predicted by early transcranial magnetic stimulation? Stroke 1996;27:2191-6.

23 Catano A, Houa M, Caroyer JM, et al. Magnetic transcranial stimulation in acute stroke: early excitation threshold and functional prognosis. Electroencephalogr Clin Neurophysiol functional progn

24 D'Olhaberriague L, Gamissans JE, Marrugat J, et al. Transcranial magnetic stimulation as a prognostic tool in stroke. F Neurol Sci 1997;147:73-80.

25 Heald A, Bates D, Cartlidge NEF, et al. Longitudinal study of central motor conduction time following stroke. Brain 1993;116:1371-85.

26 van Rijckevorsel-Harmant K, Boon V, de Barsy T. Central magnetic stimulation, somatosensory potentials, and clinical evolution during a rehabilitation treatment in hemiplegic patients. Electroencephalogr Clin Neurophysiol 1993; S102.

27 Catano A, Houa M, Caroyer JM, et al. Magnetic transcranial stimulation in non-haemorrhagic sylvian strokes: interest of facilitation for early functional prognosis. Electroencephalogr Clin Neurophysiol 1995;97:349-54.

28 Dominkus M, Grisold W, Jelinek V. Transcranial electrical motor evoked potentials as a prognostic indicator for motor recovery in stroke patients. F Neurol Neurosurg Psychiatry 1990;53:745-8

29 Zgur T, Prevec TS, Goljar N. Correlation of motor evoked potentials to motor deficit during the recovery of ischaemic stroke. Electroencephalogr Clin Neurophysiol 1993;S102.

30 Fugl-Meyer AR, Jääskö L, Leyman I, et al. The post-stroke hemiplegic patient: I. A method for evaluation of physical performance. Scand $\mathcal{F}$ Rehabil Med 1975;7:13-31.

31 Cracco RQ. Evaluation of conduction in central motor pathways: techniques, pathophysiology, and clinical interpretation. Neurosurgery 1987;20:199-203.

32 Ashworth B. Preliminary trial of carisoprodol in multiple sclerosis. Practitioner 1964;192:540-2.

33 Wade DT, Collin C. The Barthel ADL index: a standard measure of physical disability? Int Disabil Stud 1988;10:647.

34 Sanford J, Moreland J, Swanson LR, et al. Reliability of the Fugl-Meyer assessment for testing motor performance in patients following stroke. Phys Ther 1993;73:447-54.

35 Sloan RL, Sinclair E, Thompson J, et al. Inter-rater reliability of the modified Ashworth scale for spasticity in hemiplegic patients. Int F Rehabil Res 1992;15:158-61.

36 Loewen SC, Anderson BA. Reliability of the modified motor assessment scale and the Barthel index. Phys Ther 1988;68:1077-81

37 De Weerdt WJG, Harrison MA. Measuring recovery of arm-hand function in stroke patients: a comparison of the Brunnström-Fugl-Meyer test and the action research test. Physiotherapy Canada 1985;37:65-70.

38 Chiappa KH. Short-latency somatosensory evoked potentials: methodology. In: Chiappa Kh, ed. Evoked potentials in clinical medicine. Philadelphia: Lippincott-Raven, 1997:283-339.

39 Eisen AA, Shtybel W. Clinical experience with transcranial magnetic stimulation. Muscle Nerve 1990;13:995-1011.

40 Freund RJ, Litell RC. SAS system for regression. Cary, NC: SAS Institute, 1991.

41 Feys HM, De Weerdt WJ, Selz BE, et al. Effect of a therapeutic intervention for the hemiplegic upper limb in the acute phase after stroke. A single-blind, randomized, controlled multicenter trial. Stroke 1998;29:785-92. 